\title{
Synthesizing Configuration File Specifications with Association Rule Learning*
}

\author{
MARK SANTOLUCITO, Yale University, USA \\ ENNAN ZHAI, Yale University, USA \\ RAHUL DHODAPKAR, MongoDB, USA \\ AARON SHIM, Microsoft, USA \\ RUZICA PISKAC, Yale University, USA
}

System failures resulting from configuration errors are one of the major reasons for the compromised reliability of today's software systems. Although many techniques have been proposed for configuration error detection, these approaches can generally only be applied after an error has occurred. Proactively verifying configuration files is a challenging problem, because 1) software configurations are typically written in poorly structured and untyped "languages", and 2) specifying rules for configuration verification is challenging in practice. This paper presents ConfigV, a verification framework for general software configurations. Our framework works as follows: in the pre-processing stage, we first automatically derive a specification. Once we have a specification, we check if a given configuration file adheres to that specification. The process of learning a specification works through three steps. First, ConfigV parses a training set of configuration files (not necessarily all correct) into a well-structured and probabilistically-typed intermediate representation. Second, based on the association rule learning algorithm, ConfigV learns rules from these intermediate representations. These rules establish relationships between the keywords appearing in the files. Finally, ConfigV employs rule graph analysis to refine the resulting rules. ConfigV is capable of detecting various configuration errors, including ordering errors, integer correlation errors, type errors, and missing entry errors. We evaluated ConfigV by verifying public configuration files on GitHub, and we show that ConfigV can detect known configuration errors in these files.

CCS Concepts: $\bullet$ Software and its engineering $\rightarrow$ Software configuration management and version control systems; Formal software verification; Formal methods;

Additional Key Words and Phrases: Configuration Files, Program Synthesis, Association Rule Learning, Configuration Verification.

ACM Reference Format:

Mark Santolucito, Ennan Zhai, Rahul Dhodapkar, Aaron Shim, and Ruzica Piskac. 2017. Synthesizing Configuration File Specifications with Association Rule Learning. Proc. ACM Program. Lang. 1, OOPSLA, Article 64 (October 2017), 20 pages. https://doi.org/10.1145/3133888

*This research was sponsored by the NSF under grants CCF-1302327 and CCF-1715387.

Authors' addresses: Mark Santolucito, Computer Science, Yale University, 51 Prospect St. New Haven, CT, 06511, USA, mark.santolucito@yale.edu; Ennan Zhai, Computer Science, Yale University, 51 Prospect St. New Haven, CT, 06511, USA, ennan.zhai@yale.edu; Rahul Dhodapkar, Computer Science, MongoDB, 51 Prospect St. New Haven, CT, 06511, USA; Aaron Shim, Computer Science, Microsoft, 51 Prospect St. New Haven, CT, 06511, USA; Ruzica Piskac, Computer Science, Yale University, 51 Prospect St. New Haven, CT, 06511, USA, ruzica.piskac@yale.edu.

Permission to make digital or hard copies of all or part of this work for personal or classroom use is granted without fee provided that copies are not made or distributed for profit or commercial advantage and that copies bear this notice and the full citation on the first page. Copyrights for components of this work owned by others than ACM must be honored Abstracting with credit is permitted. To copy otherwise, or republish, to post on servers or to redistribute to lists, requires prior specific permission and/or a fee. Request permissions from permissions@acm.org.

(C) 2017 Association for Computing Machinery.

2475-1421/2017/10-ART64

https://doi.org/10.1145/3133888

Proc. ACM Program. Lang., Vol. 1, No. OOPSLA, Article 64. Publication date: October 2017. 


\section{INTRODUCTION}

Configuration errors (also known as misconfigurations) have become one of the major causes of system failures, resulting in security vulnerabilities, application outages, and incorrect program executions [Xu et al. 2015, 2013; Xu and Zhou 2015]. In a recent highly visible case [Ryall 2015], Facebook and Instagram became inaccessible and a Facebook spokeswoman reported that this was caused by a change to the site's configuration systems. In another case, a system configuration change caused an outage of AT\&T's 911 service for five hours. During this time, 12,600 unique callers were not able to reach the 911 emergency line [Breland 2017]. These critical system failures are not rare - a software system failures study [Yin et al. 2011], reports that about $31 \%$ of system failures were caused by configuration errors. This is even higher than the percentage of failures resulting from program bugs $(20 \%)$.

The systems research community has recognized this as an important problem and many efforts have been proposed to check, troubleshoot, and diagnose configuration errors [Attariyan and Flinn 2010; Su et al. 2007; Whitaker et al. 2004; Xu et al. 2016]. However, these tools rely on analyzing the source code of the target systems or need to run the system multiple times to understand the source of the errors. The support for a static analysis style verification for configuration files is still not on the level of automated verification tools used for regular program verification [Bobot et al. 2015; Leino 2010; Piskac et al. 2014] that can preemptively detect errors.

There have been several prior efforts that attempt to proactively verify configuration files [Huang et al. 2015; Santolucito et al. 2016; Xu et al. 2016; Zhang et al. 2014]. However, state-of-the-art efforts have limitations that are impractical to meet in practice. In general, these efforts fall into two categories with respect to their limitations.

- Tools that can detect sophisticated configuration errors, e.g., ConfigC [Santolucito et al. 2016], but at the cost of scalability. However, ConfigC requires a training set of $100 \%$ correct configuration files to extract configuration rules. Such an assumption is too strong to be impractical, because existing investigation studies [Wang et al. 2004; Yin et al. 2011] have demonstrated determining or obtaining $100 \%$ correct configuration files to drive rules is almost impossible in reality.

- Tools that do not rely on any strong assumptions e.g., EnCore [Zhang et al. 2014], PCheck [Xu et al. 2016] and ConfValley [Huang et al. 2015], but cannot handle as sophisticated errors. These tools have benefit that they do not require $100 \%$ correct training sets. In particular, these efforts cannot detect more complex configuration errors, such as ordering errors, fine-grain correlations, missing entry errors.

In this paper, we propose a framework, ConfigV, for automated verification of configuration files, which mimics standard automated verification techniques: for a given configuration file, we checked if it adheres to a specification, and if there are issues we report them to the user. Our framework surmounts both of the aforementioned shortcomings present in existing tools.

There are two main obstacles to directly applying existing automatic program verification techniques to configuration files. First, a lack of specifications on the properties of configuration files makes the verification task poorly defined. There are surprisingly few rules specifying constraints on entries, even written in plain English. Since asking users to write an entire specification for configuration files is impractical and error-prone, we instead take a specification generation approach. Second, the flat structure of configuration files is only a sequence of entries assigning some value to system variables (called keywords) and provides little structural information. In particular, the keywords in configuration files are often untyped, a useful property to leverage in program verification. 
Our main task was to first derive a specification for configuration files. We first process a training set of 256 industrial configuration files. This training set of files is available online [Xu 2017]. As these files have been deployed in industry, they contain relatively few errors. This yields a mostly correct training set.

In order to learn a specification from this large set of configuration files, the first step is to translate the training set into a more structured typed representation. We then apply our learning process to learn an abundant set of rules specifying various properties that hold on the given training set. The learning process is a more expressive form of association rule learning [Agrawal et al. 1993], which is used to learn predicate relations between multiple keywords in a configuration file. The rules, in general, specify properties such as, pairwise keyword ordering (one keyword must appear before another), or numerical value inequalities (the value of one keyword should always be greater than another). This set of learned rules constitutes a specification for a correct configuration file, which are then used to efficiently check the correctness of a user's configuration files and detect potential errors. The learning process is language-agnostic and works for any kind of configuration files, though all of the files in the training set need to be of the same language (such as MySQL or HTTPD configuration files).

ConfigV detects errors that may cause total system failures, but can also find more insidious errors, such as configurations that will slow down the system only when the server load increases beyond a certain threshold. Since these runtime errors may only be triggered after some time in a deployment environment, the standard debugging techniques [Zeller 2005] of starting a system multiple times with different configuration settings will not help detect these misconfigurations.

ConfigV works by analyzing a training set of partially correct files. A file in the training set might contain several different errors, but errors only appear in a small percentage of files. We first translate those files into an intermediary typed language. ConfigV then learns rules based on the inferred types of the keywords from the training set. However, since the files might also contain errors we take this into account when learning correct rules. All learned rules are annotated with the probability of correctness. To ensure the learned rules are correct enough, ConfigV employs a graph analysis to refine the set of learned rules. It analyzes the rule graph to rank the importance and relevance of the learned rules.

We implemented ConfigV in Haskell and evaluated it on almost 1000 real-world configuration files from GitHub. We demonstrate that we are able to detect known errors, based on StackOverflow posts, in this data set. Furthermore, we find compelling evidence that our optimizations are effective For example, we introduce a probabilistic type inference system that removes 1043 false positives errors reports to reduce the total fine grained relation errors to 325 . The rule graph analysis drastically improves the ranking of importance of the error reports, which allows users to more quickly correct the most critical misconfigurations. Additionally, ConfigV scales linearly, whereas a previous tool [Santolucito et al. 2016] showed exponential slow downs on the same benchmark set.

In summary, we make the following contributions:

- We propose the automated configuration verification framework, ConfigV, that can learn specifications from a training set of configuration files, and then use the specifications to verify configuration files of interest.

- We describe the logical foundation of using association rule learning to build a probabilistic specification for configuration files.

- We analyze the learned rules to further refine the generated specification and we empirically show the usefulness of this approach.

- We implement a ConfigV prototype and evaluate it by detecting sophisticated configuration errors from real-world dataset. 


\section{MOTIVATING EXAMPLES}

In this section we illustrate capabilities of ConfigV by using several real-world misconfiguration examples. These examples are sophisticated configuration errors that were reported on StackOverflow [sta 2017], a popular forum for programmers and administrators.

Example 1: Missing Entry Errors. Many critical system outages result from the fact that an important entry was missing from the configuration file. We call such a problem a missing entry error. In a public misconfiguration dataset [Xu 2017], many misconfiguration issues were reported exactly to be missing entry errors. Below is a real-world missing entry error example [mis 2017c] when a user configures her PHP and PostgreSQL, she needs to use both pgsql. so and curl.so in the /etc/php5/conf.d/curl.ini configuration file. This is usually achieved by the following entries in the curl configuration file:

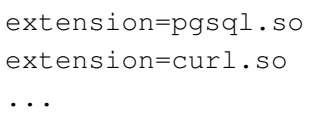

However, in this example the user accidentally left out the extension=pgsql. so entry, as done by many users [mis 2017c; Yin et al. 2011], causing a segmentation fault. If the user would run ConfigV on her file, our tool returns:

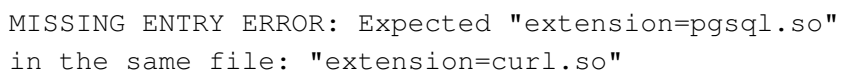

Example 2: Fine-grained Integer Correlation Errors. Our second misconfiguration example [cor 2017] comes from a discussion on StackOverflow. The user has configured her MySQL as follows:

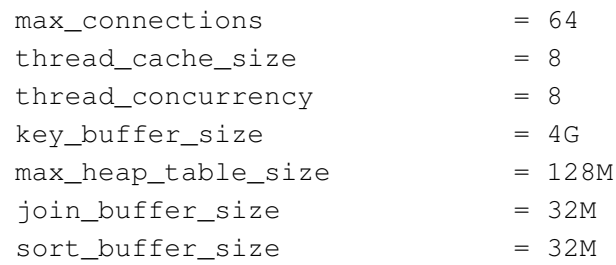

The user then complains that her MySQL load was very high, causing the website's response speed to be very slow. The accepted answer to the post reveals that the value key_buffer_size is used by all the threads cooperatively, while join_buffer and sort_buffer are created by each thread for private use. By further consulting the MySQL manual, we are instructed that when setting key_buffer_size we should consider the memory requirement of other storage engines. In a very indirect manner, we have learned that there is a correlation between key_buffer_size and other buffer sizes of the system. ConfigV learns the specific constraint that key_buffer_size should not be greater than sort_buffer_size ${ }^{*}$ max_connections. If we run ConfigV on the above configuration file, ConfigV will give an explicit answer:

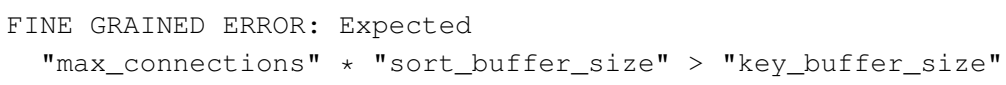

We call these errors fine-grained integer correlations. ConfigV can also detect simpler integer correlation: one entry's value should have a certain correlation with another entry's value. For instance, in MySQL, the value of key_buffer should be larger than max_allowed_packet. While several existing tools [Santolucito et al. 2016; Zhang et al. 2014] can detect simple integer correlation 


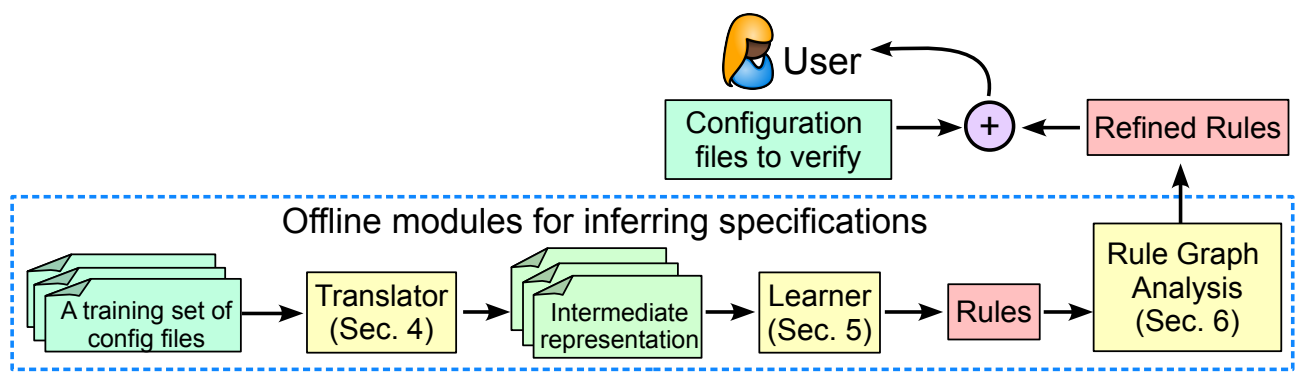

Fig. 1. ConfigV's workflow. The dashed box is the specification learning module. The yellow components are key modules of ConfigV.

errors, ConfigV is, to the best of our knowledge, the first system capable of detecting such complex fine-grained integer correlation errors.

Example 3: Type Errors. Many system availability problems are caused by assigning incorrect values of an incorrect type to a keyword. Consider the following misconfiguration file from github [typ 2017d]: a user tries to install MySQL and she needs to initiate the path of the log information generated by MySQL. This user puts the following entry assignment in her MySQL configuration file:

slow-query-log = /var/log/mysql/slow.log

This misconfiguration will lead to MySQL fails to start [que 2017]. With ConfigV, this user can get the following result:

TYPE ERROR: Expected an integer type for "slow-query-log"

This was indeed the error, since in MySQL there is another entry named "slow-query-log-file" used to specify the log path.

Example 4: Ordering Errors. Ordering errors in software configurations were first reported by Yin et al. [Yin et al. 2011], but not many existing tools can detect them. The following example contains an ordering error in a MySQL configuration file that causes the system to crash [inn 2017].

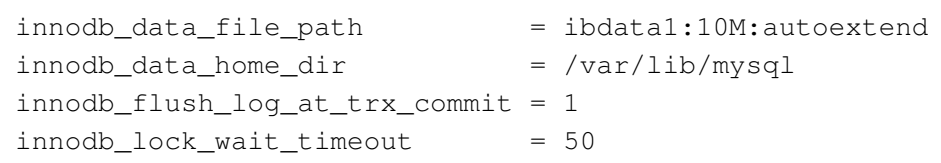

By invoking ConfigV the user receives a correct report that innodb_home_dir should appear before innodb_data_file_path, as shown below:

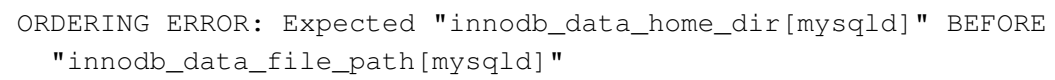

\section{THE CONFIGV FRAMEWORK OVERVIEW}

Fig. 1 gives an overview of the ConfigV framework. The main part is dedicated to learning and inferring the specification for configuration files. This process is done offline, before the user even starts to use ConfigV. There are three main steps in the process: translation, learning, and rule refinement.

Translator. The translator module first parses the input training set of configuration files and transforms them into a typed intermediate representation. Entries in a configuration file follow 
a key-value pattern, where some environmental variable ("key") is assigned a value. However, it is not always possible to fully determine the type of the key by inspecting the value at a single entry [Xu et al. 2015]. We address this problem by introducing probabilistic types. Rather than giving a variable a single type, we assign several types over a probability distribution that can later be resolved to type upon which we will learn rules.

Learning. The learner converts the intermediate representation from the translator to a set of rules. It employs a variation on the association rule algorithm [Agrawal et al. 1993], to generate this list of rules, which describe properties of a correct configuration file. This is a probabilistic verification approach that learns a specification for a correct file over an unlabeled training set of both correct and incorrect configurations files. The learning algorithm uses various instances of a rule interface to learn different classes of rules, such as ordering or integer relations. These rules are then considered necessary for any correct configuration files, and can be used for verification.

Rule Graph Analysis. Finally, the logically structured representation of learned rules allows for a further rule graph analysis. The purpose of this module is to refine the learned rules. To this end, we introduce the concept of a rule graph that can be built from the output of the modified association rule learning algorithm. We analyze the properties of this graph to construct a ranking of rules by their importance, as well as to produce a measure of complexity for any configuration of the target system. While the metrics in used in ConfigV are effective, they are not intended to be exhaustive. The information contained in the structured representation of the learned rules is a unique benefit of the learning algorithm, that has potential to be leveraged in many new ways.

\section{TRANSLATOR}

The translator takes as input a training set of configuration files and transforms it into a typed and well-structured intermediate representation. The translator can be seen as a parser used to generate an intermediate representation for the learner module (cf. Sec. 5). Parsing a file for translation is application dependent since each configuration language (MySQL, Apache, PHP) uses a different grammar. ConfigV allows users to provide extra help to the translator for their specific system configurations.

The translator converts each key-value assignment $k=v$ in the configuration file to a triple $(k, v, \tau)$, where $\tau$ is the type of $v$. There are two major challenges in this step. First is that configuration files' keywords are not necessarily unique and may have some additional context (modules or conditionals). To solve this, we rely on the fact that keywords in a configuration file must be unique within their context, and rename all keywords with their context. The set of unique keys, $\mathcal{K}$, for the sample training set in Fig. 2 would then be ["foo [server] ", "bar [client]"] .

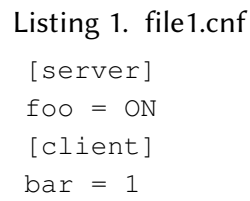

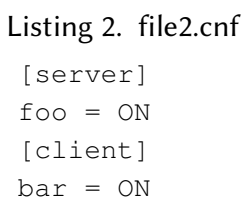

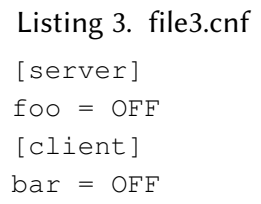

Fig. 2. A sample training set of configuration files

Probabilistic Types. An additional challenge is that it is not always possible to fully determine the type of key based on a single example value. For this reason, we introduce probabilistic types, as contrasted with basic types. In ConfigV, the finite set of basic types contains strings, file paths, integers, sizes, and Booleans. Taking the configuration 1, we can assume foo is a Boolean type by the grammar of MySQL, but the keyword bar could be many types. If we choose the type based on 


$$
\begin{aligned}
& c_{\text {int }}=|\{\forall C \in \mathcal{T} \mathcal{R} . \forall(k, v) \in C . v \in \mathbb{Z}\}| \\
& \frac{c_{\text {bool }}=\mid\{\forall C \in \mathcal{T} \mathcal{R} . \forall(k, v) \in C . v \in\{0,1, \text { ON, OFF }\}\} \mid}{k: \tilde{\tau}\left[\text { int }=c_{\text {int }}, \text { bool }=c_{\text {bool }}\right]} \text { РTYPE } \\
& \frac{k: \tilde{\tau} \quad p_{\text {int }}(\tilde{\tau})}{k:: \text { int }} \text { INT } \quad \frac{k: \tilde{\tau} p_{\text {bool }}(\tilde{\tau})}{k:: \text { bool }} \text { BooL } \\
& \frac{k_{1}:: \tau \quad k_{2}:: \tau}{e q\left(k_{1}, k_{2}\right):: \text { Rule }} \text { EQ } \quad \frac{k 1, k 2 \in C \quad k 1 \neq k 2}{\operatorname{ord}(k 1, k 2):: \text { Rule }} \text { ORDER }
\end{aligned}
$$

Fig. 3. Type judgments for a probabilistic type system with $\mathcal{T}=\{$ bool, int $\}$ and an equality and ordering rule

the first example, bar will be a integer type. If we choose a type that fits all examples, bar will be a string. However the correct classification needed is a Boolean type. For illustrative purposes, let us assume that there is an important rule we must learn about this configuration language, where two Boolean keywords should always have the same values, e.g. eq(foo, bar). If we take bar:: int, we do not learn the above rule, nor do we learn this rule with bar:: string - only with bar:: bool is the rule is valid. To resolve this ambiguity, and choose the best type, the translator assigns a distribution of types to a keyword based on examples from the training set of configuration files (denoted $\mathcal{T} \mathcal{R}=\{C\}$ ).

A probabilistic type is a set of counts over a set $\mathcal{T}$ of basic types. Formally, we define a space of probabilistic types $\tilde{\mathcal{T}}$, where $\tilde{\tau} \in \tilde{\mathcal{T}}$ has the form $\tilde{\tau}=\left\{\left(\tau_{1}, c_{1}\right), \ldots,\left(\tau_{n}, c_{n}\right)\right\}$, such that $\tau_{i} \in \mathcal{T}$, $c_{i} \in \mathbb{Z}$. Fig. 3 provides a calculus for probabilistic types over an example subset of basic types. This allows us to give a clear definition of the set of possible (i.e.well-typed) equality rules. In a general sense, probabilistic types can be seen as discrete probability distributions over basic types weighted by occurrence.

Every unique keyword $k \in \mathcal{T} \mathcal{R}$ has a probabilistic type, expressed $k: \tilde{\tau}$, as opposed to the basic type notation $k:: \tau$. The count for $\left(\tau_{i}, c_{i}\right) \in \tilde{\tau}$ should be equal to the number of times a key in $\mathcal{T} \mathcal{R}$ has a potential match to type $\tau_{i}$. The judgment PTYPE counts, over all files $C \in \mathcal{T} \mathcal{R}$, the times the value of a key matches a user defined set of acceptable values for each type $\tau_{i} \in \tilde{\tau}$. We use the notation $\tilde{\tau}\left[\tau_{i}=N\right]$ to create a probabilistic type with the count of $N$ for $\tau_{i} \in \tilde{\tau}$.

In the Bool judgment, a keyword with a probabilistic type $k: \tilde{\tau} \in \tilde{\mathcal{T}}$ can be resolved to the basic type bool when the $\tilde{\tau}$ satisfies the resolution predicate $p_{b o o l}$, i.e. the probabilistic type has sufficient evidence. The definition of sufficient evidence must be empirically determined by the user depending on the quality of the training set.

In order to define resolution predicates, we use the notation $\left|\tau_{i \tau}\right|$ to select $c_{i}$ from a $\tilde{\tau} \in \tilde{\mathcal{T}}$. As an example, for the sample training set provided in Fig. 2, we might choose to set $p_{\text {bool }}(\tilde{\tau})=\left|\operatorname{bool}_{\tilde{\tau}}\right| \geq$ $3 \wedge \mid$ int $_{\tilde{\tau}} \mid \leq 1$ and $p_{\text {int }}(\tilde{\tau})=\mid$ int $_{\tilde{\tau}} \mid \geq 3$. We then can run inference on that sample training set to derive a probabilistic type bar: [ $b o o l=3$, int $=1]$. This is then resolved, as required in our earlier example, to bar:: bool.

Note that a user may pick predicates for probabilistic type resolution that result in overlapping inference rules. For example, if a user instead picked $p_{\text {int }}(\tilde{\tau})=\left|i n t_{\tilde{\tau}}\right| \geq 1$, this predicate would overlap with the $p_{b o o l}$ predicate. This means that basic types are not unique in this system: $k::$ $\tau \wedge k:: \tau^{\prime} \nRightarrow \tau=\tau^{\prime}$.

In the case that there is not enough evidence to resolve a probabilistic type to a basic type, no type-dependent rules may be learned over that keyword. However, we are still able to learn rules such as ORDER, which do not require any resolved type. 


\section{LEARNER}

The goal of the learner is to derive rules from the intermediate representation of the training set generated by the translator. We describe an interface to define the different classes of rules that should be learned. Each instance of the interface corresponds to a different class of configuration errors, as described in Sec. 2.

To learn rules over sets of configuration files, we use a generalization of association rule learning [Agrawal et al. 1993], a technique very briefly summarized as inductive machine learning. Association rule learning is used to learn how frequently items of a set appear together. For example, by examining a list of food store receipts, we might learn that when a customer buys bread and peanut butter, the set of purchased items is also likely to include jelly. Since configuration files have multiple complex relations, we extend these association relationships to generalized predicates.

In traditional association rule learning, rules take the form of an implication:

$$
r=\left\{S_{0}, \ldots, S_{|S|}\right\} \in \text { valid } \Rightarrow\left\{T_{0}, \ldots, T_{|T|}\right\} \in \text { valid }
$$

where $S$ and $T$ are source and target sets of words. This rule states that if the set of words $S$ appear in some valid (i.e. non-rule breaking) file, the words $T$ must also appear in that file. We generalize this relation so that a rule, $r$, instead takes the following form:

$$
r=\left[S_{0}, \ldots, S_{|S|}\right] \in C_{\text {valid }} \Rightarrow C_{\text {valid }} \vdash p\left(\left[S_{0}, \ldots, S_{|S|}\right],\left[T_{0}, \ldots, T_{|T|}\right]\right)
$$

This rule relates the keywords $S_{i}, T_{j} \subseteq \mathcal{K}$ for $0 \leq i \leq|S|, 0 \leq j \leq|T|$ with a predicate $p$. Whenever the set of keywords $S$ is present in some valid configuration file, $C_{\text {valid }}$, the predicate $p$ must hold over $S$ and $T$ given the context of $C_{\text {valid }}$. In keeping with vocabulary from association rule learning, we refer to $S_{r}$ and $T_{r}$ as the source and target keyword lists of rule $r$ respectively. A rule states that if the source list of keyword appears in a valid configuration file, the given predicate must hold between the source and target lists. For clarity in notation, we define all predicates to have arity 2 by separating the source and target keywords into lists of length $|S|$ and $|T|$ respectively, but these can be equivalently thought of as predicates of arity $|S|+|T|$ as notated in Fig. 3.

Taking the food store example, our learned rule would be:

$$
[\text { bread,butter }] \in \text { valid } \Rightarrow \text { valid } \vdash \text { assoc }([\text { bread,butter }],[j e l l y])
$$

where the assoc predicate denotes that the words must all appear in a file. In this way, our formalism is more expressive than the classic association rule learning problem.

The set $\mathcal{K}$ is the set of unique keys from the training set (denoted $\mathcal{T} \mathcal{R}$ ) and the predicate $p$ is one of the classes of configuration errors. The task of the learning algorithm is to transform a training set to a set of rules, weighted with support and confidence. The set of rules learned from training set $\mathcal{T} \mathcal{R}$ constitutes a specification for a configuration file to be considered correct.

The two metrics, support and confidence, are used in association rule learning, as well as other rule based machine learning techniques [Han et al. 2007; Langley and Simon 1995]. We use slightly modified definitions of these, stated below, to handle arbitrary predicates as rules. During the learning process, each rule is assigned a support and confidence to measure the amount and quality of evidence for the rule.

$$
\begin{gathered}
\operatorname{support}(r)=\frac{\left|\left\{C \in \mathcal{T} \mathcal{R} \mid S_{r} \cup T_{r} \subseteq C\right\}\right|}{|\mathcal{T} \mathcal{R}|} \\
\operatorname{confidence}(r)=\frac{\left|\left\{C \in \mathcal{T} \mathcal{R} \mid C \vdash p_{r}\left(S_{r}, T_{r}\right)\right\}\right|}{\operatorname{support}(r) *|\mathcal{T} \mathcal{R}|}
\end{gathered}
$$

Support is the frequency that the set of keywords in the proposed rule, $S \cup T$, have been seen in the configuration files $C$ in the training set $\mathcal{T} \mathcal{R}$. Confidence is the percentage of times the rule predicate has held true over the given keywords. In the learning process, each class of rule is 


$$
\begin{array}{cc}
\frac{k_{1}:: \text { bool }}{\text { isbool }\left(\left[k_{1}\right],\left[k_{1}\right]\right):: \text { Rule }} \text { BOoL } & \frac{k_{1}:: \text { int }}{\operatorname{isint}\left(\left[k_{1}\right],\left[k_{1}\right]\right):: \text { Rule }} \text { INT } \\
\frac{k_{1}, k_{2}:: \text { int }}{\text { missing }\left(\left[k_{1}\right],\left[k_{2}\right]\right):: \text { Rule }} \text { MIssing } & \frac{\operatorname{compare}\left(\left[k_{1}\right],\left[k_{2}\right]\right):: \text { Rule }}{\text { COARSE_GRAIN }} \\
\frac{k_{1}, k_{2}, k_{3}:: \text { int }}{\text { compare }\left(\left[k_{1}, k_{2}\right],\left[k_{3}\right]\right):: \text { Rule FINE_GRAIN }} & \frac{k_{1}, k_{3}:: \text { size } \quad k_{2}:: \text { int }}{\text { compare }\left(\left[k_{1}, k_{2}\right],\left[k_{3}\right]\right):: \text { Rule FINE_GRAIN }}
\end{array}
$$

Fig. 4. An expanded set of typing judgements for valid rules

manually assigned a support and confidence threshold, $t_{s}$ and $t_{c}$ respectively, below which a rule will be rejected for lack of evidence. We denote the set rules that are learned and included as part of the final specification are as follows:

$$
\begin{gathered}
\operatorname{Learn}(\mathcal{T R})=\left\{r \mid \text { support }(r)>t_{s} \wedge\right. \\
\text { confidence }(r)>t_{c} \wedge \\
S, T \subseteq \mathcal{K}\}
\end{gathered}
$$

\subsection{Error Classes}

Each class of error forms a rule with a predicate $p$, and choices of $|S|$ and $|T|$, the sizes of the source and target keyword lists. Ordering errors require $|S|=1,|T|=1$ and use the predicate order which means if both keywords $S_{0}, T_{0}$ appear in a file, $S_{0}$ must come before $T_{0}$. For example, the ordering error given in Sec. 2 is expressed:

$$
\begin{aligned}
& \text { [innodb_data_home_dir] } \in C_{\text {valid }} \Rightarrow \\
& \qquad C_{\text {valid }} \vdash \operatorname{order}([\text { innoddb_data_home_dir], [innodb_data_file_path]) }
\end{aligned}
$$

Missing keyword entry errors require $|S|,|T|=1$ and use the predicate missing to mean the keyword $T_{0}$ must appear, in any location, in the same file as the keyword $S_{0}$ in any configuration file. The type rule is a set of rules over multiple predicates, where $|S|=1,|T|=1$. These type rules take the form $S_{0} \in C_{\text {valid }} \Rightarrow C_{\text {valid }} \vdash$ is_( $\left.\left[S_{0}\right],\left[S_{0}\right]\right)$ where _ (underscore) matches all the basic types (bool, int, size, etc), as shown in Fig. 4. In this case, the source and target are the same keyword, since a type constraint is only on a single keyword.

ConfigV also supports two types of integer correlation rules, coarse-grained and fine-grained. Both integer correlation rules are set of rules over the set, compare, of predicates $\{<,=,>\}$. Coarse grain rules require $|S|,|T|=1$, and the predicates have the typical interpretation. Fine-grained rules use $|S|=2,|T|=1$, and interpret the predicates such that for $k_{1}, k_{2} \in S, k 1 * k 2$ must have the predicate relation to $T_{0}$. The integer correlation rules also use probabilistic types to prune the search space. To avoid learning too many false positives, we restrict this rule to either size $*$ int $=$ size, or int $*$ int $=i n t$, as shown in Fig. 4. Without probabilistic typing, we would also learn rules which do not have a valid semantic interpretation, for example a relation between three size keywords $(k 1, k 2, k 3::$ size $)$ of the form $k 1 * k 2>k 3$.

We chose the predicates in Fig. 4 based on our own experience, as well as prior issues identified in [Xu et al. 2015; Yin et al. 2011]. Other predicates might also be interesting for other configuration languages, for example comparing IP subnets. Predicates are not language dependent and we have defined the core predicates that should be needed for most configuration languages here. If a user requires other predicates for their particular use case, they may be added following the same format as above. 


\subsection{Checker}

With the rules generated by the learner module, ConfigV checks whether any entry in a target configuration file violates the learned rules and constraints. ConfigV parses a single verification target configuration file with the translator from Sec. 4 to obtain a set of key-value pairs, $C$, for that file. Then, the checker applies the learner from Eq. 1 with Learn $(C)$, to build the set of relations observed in the file, with the thresholds $t_{s}, t_{c}=100 \%$. The checker will then report the following set of errors:

$$
\begin{aligned}
\operatorname{Errors}(C)=\{r \mid & S_{r} \cup T_{r} \in C \wedge \\
& r \in \operatorname{Learn}(\mathcal{T} \mathcal{R}) \wedge \\
& r \notin \operatorname{Learn}(C)
\end{aligned}
$$

For any relation from the verification target that violates a known rule, the checker will report the predicate and keyword sets associated with that rule as an error. Since this is a probabilistic approach, in our tool ConfigV, we provide the user with the support and confidence values as well to help the user determine if the rule must be satisfied in their particular system. For instance, the key_buffer misconfiguration from Sec. 2 will only be noticeable if the system experiences a heavy traffic load, so the user may choose to ignore this error if they are confident this will not be an issue.

\section{RULE GRAPH ANALYSIS}

The learner outputs a set of rules learned from the training set as described in Sec. 5. Recall that a rule is an implication relationship of the basic form $r=S_{r} \in C_{\text {valid }} \Rightarrow C_{\text {valid }} \vdash p\left(S_{r}, T_{r}\right)$. This data is necessary to perform the core verification task, but can also be used for further analysis. By interpreting the rules as a graph (which we call the rule graph), we can use tools from graph theory to extract information about the configuration space that can improve the quality of the learned model. We inspect properties of this rule graph to sort reported errors by those most likely to be valid. To demonstrate the additional value of the rule graph, we also use it to estimate the complexity of a configuration file.

Accessibility of the rule graph is a useful property of the association rule learning technique applied by ConfigV. While it is possible to analyze the models from other machine learning techniques, such as neural networks [Lei et al. 2016] and conditional random fields [Raychev et al 2015], these analyses require a deep knowledge of the applied techniques. In contrast, the rule graph is a relatively simple, yet information rich, representation of the learned model. The following section provides a precise definition of the rule graph and demonstrates useful metrics we derive for the purposes of ranking reported errors and complexity analysis.

\subsection{Rule Ordering}

We define the rule graph as a directed hypergraph $H=(V, E)$, with vertices $V=\{k e y w o r d s\}$ and labeled, weighted edges $E=\left\{\left(V_{s}, V_{t}, l, w\right)\right\}$. The set of edges is constructed from the learned rules, using the source and target keyword sets as sources and targets respectively, the predicates as labels, and the confidence as weights:

$$
\begin{aligned}
& \forall r \in \operatorname{Learn}(\mathcal{T} \mathcal{R}) . \exists e \in E . \\
& \quad V_{s}=S_{r} \wedge V_{t}=T_{r} \wedge l=p_{r} \wedge w=\text { confidence }(r)
\end{aligned}
$$




$$
\begin{array}{r}
\text { type }_{I N T}(\{k 1\},\{k 1\}) \\
\text { missing }(\{k 1\},\{k 2\}) \\
\text { order }(\{k 2\},\{k 3\}) \\
\text { fine grain }_{\geq}(\{k 1, k 3\},\{k 2\}) \\
\mathcal{D}(k 1)=0.9+0.9+0.8+0.6
\end{array}
$$

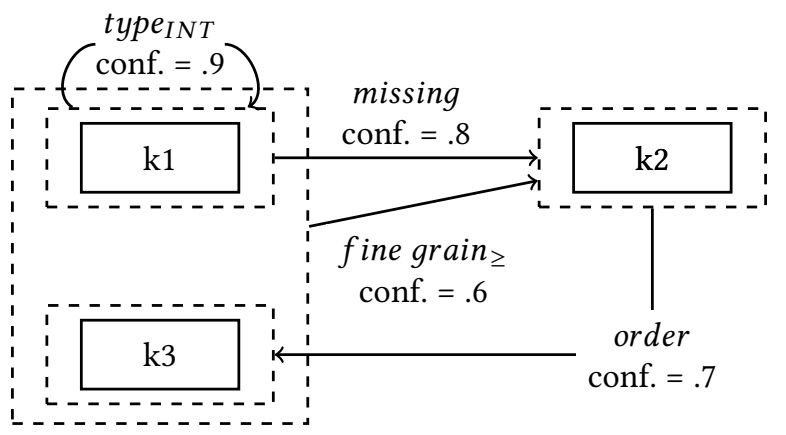

Fig. 5. A rule graph constructed from the example rules over keywords k1, k2, k3 on the left. Dashed lines indicate the source and target vertex sets for each rule. The rules type $I N T$, missing, and fine grain $\geq$ are in the slice sets used to calculate the degree of $\mathrm{k} 1$.

We will also denote $E_{V_{1}, V_{2}} \subset E$ as the slice set of $E$ over $V_{1}, V_{2}$. We can think of $E_{V_{1}, V_{2}}$ as the subset of edges in $E$ such that each source set $V_{s}$ shares a vertex with $V_{1}$ and each target set $V_{t}$ shares a vertex with $V_{2}$. Formally:

$$
\begin{array}{r}
E_{V_{1}, V_{2}}=\left\{\left(V_{s}, V_{t}, l, w\right) \in E \mid \exists v_{1} \in V_{1} \wedge v_{1} \in V_{s} \wedge\right. \\
\left.\exists v_{2} \in V_{2} \wedge v_{2} \in V_{t}\right\}
\end{array}
$$

We denote a standalone vertex $v$ in our subscripts as notational convenience for the singleton set containing that vertex $v$.

The size of an edge set, $|E|$, is the sum of all weights in that set. The use of the support and confidence thresholds $t_{s}$ and $t_{c}$ in the learner ensure that all weights in the rule graph are positive. We then define a measure of degree, $\mathcal{D}(v)$, for each vertex $v$ as the sum of in-degree and out-degree.

$$
\begin{gathered}
|E|=\sum_{(S, T, l, w) \in E} w \\
\mathcal{D}(v)=\sum_{v^{\prime} \in V}\left|E_{v, v^{\prime}}\right|+\sum_{v^{\prime} \in V}\left|E_{v^{\prime}, v}\right|
\end{gathered}
$$

As an example of the rule graph construction and degree calculation, consider the rules presented in Fig.5. We assume that those rules are the result of learning over some training set, and have confidence values as notated in the graph. We can then construct the rule graph as demonstrated on the right of Fig. 5. The rule graph is then used to calculate the degree of the keywords, which indicate the estimated importance of each keyword.

We may now use this measure to rank our errors. The more rules of high confidence are extracted for a keyword by the learner, the higher the $\mathcal{D}(v)$ of the corresponding vertex in the rule graph. In our final analysis, we use this classification of rules to order the reported errors by estimated importance.

Keywords (specifically their corresponding vertices) of low $\mathcal{D}(v)$ may be rarer configuration parameters where rules learned are more likely to be governed by technical necessity, rather than industry convention. As such, errors reported involving low-degree keywords are more likely to be errors of high significance and should be presented with high importance to users of ConfigV.

Specifically, for an error reported by ConfigV on a rule $r$ involving keywords $K$, we rank the errors by: 


$$
\operatorname{RANK}(r)=\frac{\sum_{k \in K} \mathcal{D}(k)}{|K|}
$$

The results from ranking errors in this way are presented in Sec. 7.

\subsection{Complexity Measure}

We may also use the rule graph to advance our general knowledge of the configuration space, outside the strict confines of a verification system. As an example, we present a heuristic for configuration file complexity based on the topology of the rule graph. This measure of complexity could be used by software organizations to manage configuration files in much the same way as Kolomogrov complexity [Kolmogorov 1965] is used to manage code - identifying potentially brittle configurations for targeted refactoring.

For a configuration file with a set of keywords $K$ and a rule graph $H=(V, E)$, we define our complexity measure:

$$
C(K, H)=\sum_{k \in K} \begin{cases}1 *\left(1-\frac{\left|E_{k, K}\right|}{\left|E_{k, V}\right|}\right) & \text { if }\left|E_{k, V}\right|>0 \\ 1 & \text { otherwise }\end{cases}
$$

The complexity measure, $C$, can be thought of as an extension of the naïve line-counting measure of complexity. When a keyword in the configuration file is present in the rule graph, we may consider the set $E_{k, K}$ to be all learned rules involving keyword $k$ that are relevant to the configuration file being examined. The set $E_{k, V}$ denotes all learned rules involving $k$. Given these sets, we may think of $\frac{\left|E_{k, K}\right|}{\left|E_{k, V}\right|}$ as representing the amount that $k$ is constrained in the current configuration file relative to how much it could be constrained in the global configuration space. The more constrained a configuration keyword in a particular configuration file, the less it should contribute to the complexity (hence $1 *\left(1-\frac{\left|E_{k, K}\right|}{\left|E_{k, V}\right|}\right)$ ). If a keyword is not constrained at all in the current configuration file or is not present in the rule graph, we revert to the standard counting metric of complexity.

While an in-depth evaluation of the complexity metric presented here is out of scope for this paper, we use this measure to demonstrate the flexibility of the rule graph, and potential for further applications.

\section{IMPLEMENTATION AND EVALUATION}

We have implemented a tool, ConfigV, and evaluated it based on real-world configuration files taken from GitHub. ConfigV is written in Haskell and is available open source at https://github. $\mathrm{com} /$ santolucito/configV. Thanks to Haskell's powerful type system, the implementation can easily be extended with new rule classes or applied to different configuration languages with minimal change to the rest of the code base. When adapting ConfigV to a new language, the key components that must be provided are a parser into our intermediate representation and a training set of configuration files in the new language. In some cases, a user may also require new types or predicates to learn over. In this case, a user only needs to provide the functions for the rule interface (a typeclass in Haskell) to 1) learn relations from a single file 2) merge two sets of rules and 3) check a file given some set of rules.

\subsection{Evaluation}

To evaluate our ConfigV prototype, we require a separate training set and test set. For the training set, $\mathcal{T} \mathcal{R}$, we use a preexisting set of 256 industrial MySQL configuration files collected in previous configuration analysis work [Xu 2017]. This is an unlabeled training set, though most of the files have some errors. For the test set, we randomly sampled 1000 MySQL configuration files from 
GitHub, and filtered the incorrectly formatted files out for a final total of 973 files. While our tool can detect incorrectly formatted files, we exclude them here as they do not add any useful information to the training set. The focus of our evaluation are MySQL files for comparability of results, but ConfigV can handle any configuration language (that can be parsed to the intermediate representation from Sec. 4).

We report the number of rules learned from the training set and the number of errors detected in the test set in Table 1. One interesting note is that without probabilistic types we learned 327 fine grained rules and detected 1368 fine grained errors. By introducing probabilistic types, we remove 224 incorrect rules and thereby remove 1043 false positives. We are guaranteed these are all false positives since there cannot be a correct rule of an incoherent type. For example, comparing the types size * size and size is not meaningful, and thus cannot be a correct rule, because of the semantic interpretation of the size units as memory.

We also provide the support and confidence thresholds, $t_{s}, t_{c}$, used in this evaluation. These thresholds can be adjusted by the user to control the level of assurance that their file is correct. The ideal setting will depend on both the user preference and training set quality. In our evaluation, we adjust the support and confidence values such that when ConfigV is run on a file with no more than 3 known errors, ConfigV reports those errors and no others. Following common practice from association rule learning, initial values for support are confidence are generally in the range of $10 \%$ and $90 \%$ respectively.

We record the histogram of errors across the test set in Fig. 6. This is intuitively an expected result from randomly sampling GitHub - most repositories will have few errors, with an increasingly small number of repositories having many errors. When sampling repositories from GitHub, we searched for any files with the common MySQL extension '.cnf'. To ensure the files we found were really configurations for MySQL we searched specifically for files that contained keywords common to MySQL such as 'mysql' and 'size' and 'innodb'. The repository sampling had a wide variety of sizes and contributors. The average number of contributors per repository was 5.85 with a standard deviation of 9.73 . The average number of commits per repository was 2767.04 with a standard deviation of 10362.57 . These numbers indicate the wide range of repositories sampled.

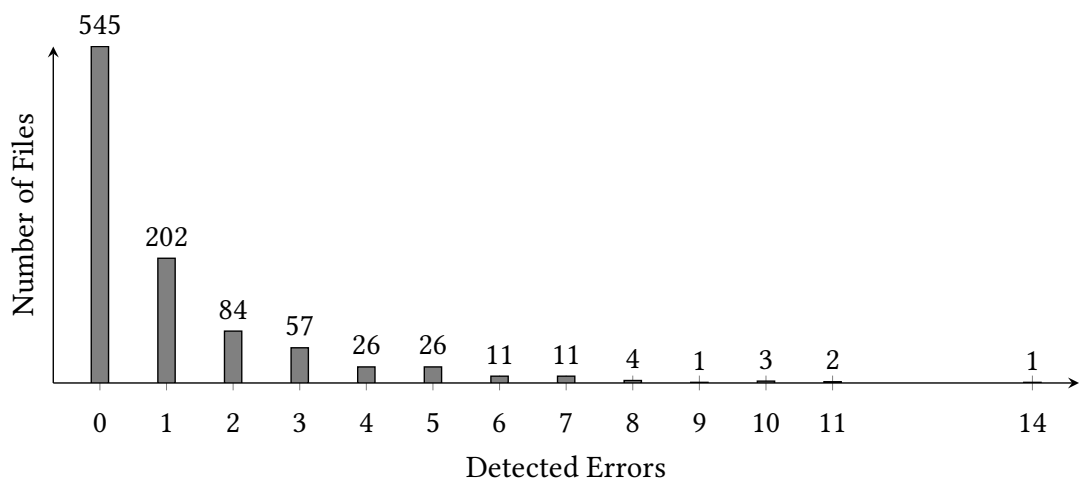

Fig. 6. Histogram of errors

The errors reported over the GitHub files may have varying impacts on the system, ranging from failing to start, runtime crash, or performance degradation. However, since ConfigV is a probabilistic system, it is also possible that some errors are false positives - that is, a violation of the rule has no effect on the system. Note that in contrast to program verification, we do not have an oracle for 
Table 1. Results of ConfigV

\begin{tabular}{|c|c|c|c|c|}
\hline Class of Error & \# Rules Learned & \# Errors Detected & Support & Confidence \\
\hline \hline Order & 12 & 63 & $6 \%$ & $94 \%$ \\
Missing & 53 & 55 & $2 \%$ & $71 \%$ \\
Type & 94 & 345 & $12 \%$ & $70 \%$ \\
Fine-Grain & 103 & 325 & $24 \%$ & $91 \%$ \\
Coarse-Grain & 97 & 237 & $10 \%$ & $96 \%$ \\
\hline
\end{tabular}

determining if a reported error is a true error or a false positive. While we can run a program to determine the effect a specification has on the success of compiling/running the program, no such test exists for configuration files. Because configurations are dependent on the rest of the system (i.e., the available hardware, the network speed, and the usage patterns), we cannot simulate all the conditions to determine if a reported error will cause system failure. As evidenced by Example 2, some misconfigurations will only cause greater than expected performance degradation, and only under particular traffic loads. In light of this, the definition of a true error is necessarily imprecise.

\subsection{Issue Reporting Back to Users}

As one metric to help evaluate the usefulness of our tool, we submitted GitHub issue reports for these bugs. Too avoid unnecessary burden on these users, we reported only at most the top three issues for each configuration file. Additionally, we submitted at most one report per user, so that users with multiple configuration files that we tested would receive a single message. Applying these filters resulted in a total of 215 issue reports submitted to open source repositories - the issues can be found at through the GitHub search at https://goo.gl/KNFuuz. At the time of writing this paper, we have received feedback on 21 of these issues. Because of the variety of feedback, we present a selection of the responses from users.

An interesting point is two users had opposite reactions to the same error. The error Expected query-cache-type [mysqld] <= max-allowed-packet [mysqld] was reported to two different users. While one user was appreciative and interested, replying with the message "Wow, thanks guys", the other user responded in colorful language that he does not believe that these two values should even be compared. A number of users responded that they could not provide feedback because the repository was outdated or not maintained and they were not sure of the effect such an error might have on the system.

We also received feedback that helped us discover some limitations of our training set and pointed us towards future improvements. For example, when we reported the error Expected "key-buffer [isamchk]" in the same file as "[isamchk]", the user commented that key-buffer is a depreciated keyword that has been replaced with key-buffer-size. In this case, our training set of files had been used to configuration an earlier version of MySQL than the target verification file of the user. The error in this case is in fact a true positive for earlier versions of MySQL, but in later versions, key-buffer-size is preferred instead.

While this use case highlighted a limitation of our training set, it also provides further motivation that such a tool is important. As configuration specifications are constantly changed, maintaining a manually written set of specification is an increasingly impractical task. We have learned from this case study that, in practice, it will be important to ensure the training set of is using the same, or similar, system version as the verification target. 
Table 2. Sampled misconfiguration files for error detection evaluation.

\begin{tabular}{|c|c|c|c|c|c|}
\hline Errors & URLs & None & RG & PT & RG^PT \\
\hline \multirow{3}{*}{$\begin{array}{l}\text { ORDERING ERROR: Expected “innodb_data_home_dir" BEFORE } \\
\text { "innodb_data_file_path" }\end{array}$} & [ord 2017c] & $12 / 12$ & $3 / 12$ & $5 / 5$ & $3 / 5$ \\
\hline & [ord 2017a] & $11 / 11$ & $2 / 11$ & $3 / 3$ & $3 / 3$ \\
\hline & [ord 2017b] & $9 / 9$ & $3 / 9$ & $4 / 4$ & $3 / 4$ \\
\hline \multirow{3}{*}{ MISSING ERROR: Expected “key_buffer” WITH [isamchk] } & [mis 2017d] & $6 / 10$ & $2 / 10$ & $2 / 4$ & $2 / 4$ \\
\hline & [mis 2017a] & $2 / 3$ & $3 / 3$ & $2 / 3$ & $3 / 3$ \\
\hline & [mis 2017b] & $2 / 3$ & $3 / 3$ & $2 / 2$ & $3 / 3$ \\
\hline \multirow{3}{*}{ TYPE ERROR: Expected bool for "thread_cache_size" } & [typ 2017c] & $1 / 12$ & $1 / 12$ & $1 / 4$ & $1 / 4$ \\
\hline & [typ 2017a] & $1 / 9$ & $1 / 9$ & $1 / 1$ & $1 / 1$ \\
\hline & [typ 2017b] & $1 / 8$ & $1 / 8$ & $1 / 3$ & $1 / 3$ \\
\hline \multirow{3}{*}{$\begin{array}{l}\text { FINE GRAINED ERROR: Expected } \\
\text { "max_connections" * "sort_buffer_size" > "key_buffer_size" }\end{array}$} & [fin 2017a] & $30 / 34$ & $18 / 34$ & $6 / 7$ & $3 / 7$ \\
\hline & [fin 2017c] & $23 / 25$ & $9 / 25$ & $8 / 9$ & $3 / 9$ \\
\hline & [fin 2017b] & $20 / 23$ & $14 / 23$ & $6 / 7$ & $5 / 7$ \\
\hline \multirow{3}{*}{$\begin{array}{lccc}\text { INTEGER } & \text { CORRELATION } & \text { ERROR: } & \text { Expected } \\
\text { "max_allowed_packet" < "innodb_buffer_pool_size" } & \end{array}$} & [int 2017c] & $29 / 32$ & $8 / 32$ & $11 / 14$ & $4 / 14$ \\
\hline & [int 2017a] & $22 / 23$ & $2 / 23$ & $9 / 10$ & $2 / 10$ \\
\hline & [int 2017b] & $10 / 12$ & $4 / 12$ & $4 / 4$ & $2 / 4$ \\
\hline
\end{tabular}

\subsection{Sorting Error Reports}

Although we cannot identify false positives, we can identify true positives by examining online forums, like StackOverflow. On these forums we find reports that particular configuration settings have caused problems on real-world systems. Furthermore, any error for which we can find evidence online is likely to be more problematic than errors that do not have an online record, using the reasoning that this error has caused enough problems for people to seek help online. Given that some errors are more severe than others, we would like ConfigV to sort the errors by their importance or potential severity. To achieve this sorting we use the rule graph analysis metric described in Sec. 6.1.

To estimate the impact of this metric, we track the rank of known true positives with, and without, the augmented rule ordering in Table 2. For this table, we picked the known true positive rules, listed in the Errors column, and pick configuration files in the test set that have these errors. We picked 3 files for each true positive by choosing the files with the highest number of total reported errors in order to clearly observe the effects of our optimizations. Although this gives a more clear picture of the effect of our optimizations, it results in a slightly inflated false positive rate.

We test the following conditions; just rule graph analysis (RG) to sort the errors, just probabilistic types to filter the rules $(\mathrm{PT})$, and both optimizations at the same time $(\mathrm{RG} \wedge \mathrm{PT})$. For each entry we list $\mathrm{X} / \mathrm{Y}$, where $\mathrm{X}$ is the rank of the known true positive, and $\mathrm{Y}$ is the total number of errors found in that file.

\subsection{False Positive Rate}

Because ConfigV detects complex and subtle misconfigurations that, for example, may cause performance degradation in a high traffic load, false positives are system and use-case dependent and therefore ill-defined. However, we report an estimation of the false positive rate for comparison to other tools. To estimate a false positive rate, we asked two industry experts, one from MongoDB and one from Microsoft, to independently classify all errors from Table 2. For each unique error reported in Table 2 (a total of 70 unique errors), the expert was asked to classify the error as: definitely false positive, potential true positive, or definitely true positive. The MongoDB expert rated 13/70 errors as definitely false positives. The Microsoft expert rated 8/70 errors as definitely 
Table 3. Time for training over various training set sizes

\begin{tabular}{|c|c|c|}
\hline \# of Files for Training & ConfigC (sec) & ConfigV (sec) \\
\hline \hline 0 & 0.051 & 0.051 \\
\hline 50 & 1.815 & 1.638 \\
\hline 100 & 13.331 & 4.119 \\
\hline 150 & 95.547 & 10.232 \\
\hline 200 & 192.882 & 12.271 \\
\hline 256 & 766.904 & 15.627 \\
\hline
\end{tabular}

false positives. The similarity between experts is suggestive that these are approximately correct classifications.

The resulting false positive rate is then estimated to be $11 \%-18 \%$. This is within the range of the false positive rate in existing work. For example in the EnCore tool, which had a false positive rate of $13 \%, 21 \%, 32 \%$ for MySQL, Apache, and PHP respectively [Zhang et al. 2014]. We note again that as opposed to a tool like EnCore, which is used mainly to detect initialization errors, thanks to the complex relations that can be learned, ConfigV also learns misconfigurations causing runtime performance degradation. This means ConfigV generates a larger rule set, and false positives cannot be guaranteed, i.e. there may be some environment conditions that will cause a "false" positive to become a true positive.

In contrast, a true positive can be confirmed as such based on evidence of unwanted system behavior. The errors listed in Table 2 are confirmed true positives, evidenced by posts on help forums. ConfigV detected and reports these errors in the 15 code repositories listed in the URL column of Table 2 . These are real-world configuration files that contain errors that may be unknown to the maintainers of the repositories.

\subsection{Runtime Performance}

We also evaluate the speed of ConfigV. Generally, once a set of rules has been learned, it is not necessary to rerun the learner. However, using ConfigV on a new language does require rerunning the learner. We have only used ConfigV to build rules for MySQL here, but any configuration language might be analyzed with ConfigV given a parser and training set. Additionally, in an industrial setting, the available training set may be much larger than ours, so it is important that the learning process scales. We see in Table 3 that ConfigV scales roughly linearly.

We compare ConfigV to our prior work in configuration verification, ConfigC [Santolucito et al. 2016]. Although ConfigC does not support as advanced features as ConfigV, such as fine-grained correlations, probabilistic types, or rule graph ordering, we report training times here for both tools over the same training set on configuration files. ConfigC scales exponentially because the learning algorithm assumes a completely correct training set, and learns every derivable relation. With ConfigV, we instead only process rules that meet the required support and confidence, reducing the cost of resolving to a consistent set of rules. The times reported in Table 3 were run on four cores of a Kaby Lake Intel Core i7-7500U CPU @ 2.70GHz on 16GB RAM and Fedora 25. Even with support for the more advanced features, ConfigV still far outperforms ConfigC.

\section{RELATED WORK}

Configuration verification has been considered a promising way to tackle misconfiguration problems [Xu and Zhou 2015]. Nevertheless, a practical and automatic configuration verification approach still remains an open problem. 
Language-support misconfiguration checking. There have been several language-support efforts proposed for preventing configuration errors introduced by fundamental deficiencies in either untyped or low-level languages. For example, in the network configuration management area, administrators often produce configuration errors in their routing configuration files. PRESTO [Enck et al. 2007] automates the generation of device-native configurations with configlets in a template language. Loo et al. [Loo et al. 2005] adopt Datalog to reason about routing protocols in a declarative fashion. COOLAID [Chen et al. 2010] constructs a language to describe domain knowledge about devices and services for convenient network reasoning and management. Compared with the above efforts, ConfigV mainly focuses on software systems, e.g., MySQL and Apache, while our main purpose is to automate configuration verification rather than proposing new languages to convenient configuration-file writing.

The closest effort to ConfigV is ConfigC [Santolucito et al. 2016], which aims to learn configurationchecking rules from a given training set. Compared with ConfigV, ConfigC has the following disadvantages. First, ConfigC requires the configuration files in the training set must be correct, which is impractical because it is very difficult to determine a correct configuration set in reality. On the contrary, since ConfigV does not rely on the quality of configuration files in training sets, ConfigV is much more practical than ConfigC. Second, ConfigC covers fewer types of misconfigurations than ConfigV. For example, ConfigC cannot detect fine-grained integer correlation errors. Finally, the training time of ConfigC is much longer than ConfigV (as shown in Table 3). Furthermore, ConfigC was originally presented only as a tool, and did not provide the theoretical framework that would allow it to be used in other applications. This paper has provided an succinct explanation additionally both for ConfigV as well as giving ConfigC a theoretical context to more accurately compare against ConfigV.

Misconfiguration detection. Misconfiguration detection techniques aim at checking configuration efforts before system outages occur. Most existing detection approaches check the configuration files against a set of predefined correctness rules, named constraints, and then report errors if the checked configuration files do not satisfy these rules. Huang et al. [Huang et al. 2015], for example, proposed a language, ConfValley, to validate whether given configuration files meet administrators' specifications. Different from ConfigV, ConfValley does not have inherent misconfiguration checking capability, since it only offers a language representation and requires administrators to manually write specifications, which is an error-prone process. On the contrary, ConfigV does not need users to manually write anything.

Several machine learning-based misconfiguration detection efforts also have been proposed [Xu et al. 2016; Yuan et al. 2011; Zhang et al. 2014]. EnCore [Zhang et al. 2014] introduces a templatebased learning approach to improve the accuracy of their learning results. The learning process is guided by a set of predefined rule templates that enforce learning to focus on patterns of interest. In this way, EnCore filters out irrelevant information and reduces false positives; moreover, the templates are able to express system environment information that other machine learning techniques cannot handle. Compared with EnCore, ConfigV has the following advantages. First, ConfigV does not rely on any template. Second, EnCore cannot detect missing entry errors, type errors, ordering errors and fine-grained integer correlation errors, but ConfigV can detect all of them. Finally, ConfigV is a very automatic system, but EnCore needs significant human interventions, e.g., system parameters and templates.

PCheck [Xu et al. 2016] aims to add configuration checking code to the system source code by emulating potential commands and behaviors of the system. This emulation is a "white-box" approach and requires access to the system's source code. One drawback to this approach is that for some systems (e.g., ZooKeeper) whose behavior is hard to emulate, PCheck cannot automatically 
generate the corresponding checking code. Due to the emulation based testing strategy, PCheck's scope is limited to reliability problems caused by misconfiguration parameters. In contrast, ConfigV is a "black-box" approach and only requires a training set of configuration files to learn rules. By using a rule learning strategy of examples, ConfigV is able to detect general misconfiguration issues that are outside the scope of emulation testing (e.g.memory or thread usage settings), including performance, security, availability and reliability.

Misconfiguration diagnosis. Misconfiguration diagnosis approaches have been proposed to address configuration problems post-mortem. For example, ConfAid [Attariyan and Flinn 2010] and X-ray [Attariyan et al. 2012] use dynamic information flow tracking to find possible configuration errors that may have resulted in failures or performance problems. AutoBash [Su et al. 2007] tracks causality and automatically fixes misconfigurations. Unlike ConfigV, most misconfiguration diagnosis efforts aim at finding errors after system failures occur, which leads to prolonged recovery time.

Association Rule Learning. The approach we have presented not only generalizes association rule learning, but also another learning strategy called sequential pattern mining [Mabroukeh and Ezeife 2010]. Ordering rules are similar to the patterns learned in sequential pattern mining, although we restrict our ordering rules to $|S|,|T|=1$ since these are the most common misconfigurations we encounter in practice. While a major limitation to sequential pattern mining is the scalability of the problem [Ayres et al. 2002], we escape this issue with $|S|,|T|=1$ and the fact that a single configuration file tends to be less a few thousand lines. There has been other work in these same classes of algorithms [Han et al. 2007; Langley and Simon 1995] for various applications and variations on the core problem. A future direction for this work is to integrate advances in these domains into the configuration file verification problem.

\section{CONCLUSION}

In this paper, we introduce ConfigV, a highly modular framework that allows automatic verification of configuration files. The main problem for verification of configuration files is their lack of specification, so they are not a traditional target area for formal methods. Inspired by the association rule learning algorithm, ConfigV learns a set of rules which describe properties and relations between keywords appearing in configuration files. These rules corresponding to the specification. Our evaluation, based on real-world examples, shows that ConfigV is able to correctly detect and report configuration errors including ordering, missing entry, integer correlation, and type errors.

\section{REFERENCES}

2017a. Aymargeddon. https://raw.githubusercontent.com/bennibaermann/Aymargeddon/ b85d23c0690b1c6a48a045ea45f4c8b19b036fa5/var/my.cnf. (March 2017).

2017a. container. https://www.dropbox.com/s/5alc0zs0qp5i529/ybh8r3n2avj7sqd1rcmx0orzry23bopl.cnf?dl=0. (March 2017).

$2017 \mathrm{a}$.

containerization.

https://raw.githubusercontent.com/billycyzhang/containerization/ 78c6e8fefbafb89de8c28296e83a2f6fefe03879/enterprise-images/mariadb/my.cnf. (March 2017).

2017a. evansims. https://raw.githubusercontent.com/evansims/scripts/715e4f4519bbff8bab5ab26a15256d79796c923a/config/ mysql/my-2gb.cnf. (March 2017).

2017b. evansims-script. https://raw.githubusercontent.com/evansims/scripts/715e4f4519bbff8bab5ab26a15256d79796c923a/ config/mysql/my-1gb.cnf. (March 2017).

2017. Fatal Error: Cannot allocate memory for the buffer pool. http://dba.stackexchange.com/questions/25165/ intermittent-mysql-crashes-with-error-fatal-error-cannot-allocate-memory-for-t. (March 2017).

2017. Fine-grained value correlation error. (March 2017). http://serverfault.com/questions/628414/ my-cnf-configuration-in-mysql-5-6-x. 
2017b. isucon2-summer-ruby. https://raw.githubusercontent.com/co-me/isucon2-summer-ruby/ 1f633384f485fb7282bbbf42f2bf5d18410f7307/config/database/my.cnf. (March 2017).

2017b. mini-2011. https://raw.githubusercontent.com/funtoo/experimental-mini-2011/ 083598863a7c9659f188d31e15b39e3af0f56cab/dev-db/mysql/files/my.cnf. (March 2017).

2017c. mysetup. https://raw.githubusercontent.com/kazeburo/mysetup/99ba8656f54b1b36f4a7c93941e113adc2f05f70/mysql/ my55.cnf. (March 2017).

2017c. PHP CLI Segmentation Fault with pgsql. http://linux.m2osw.com/php_cli_segmentation_fault_with_pgsql. (March 2017).

$2017 \mathrm{~b}$

puppet. https://raw.githubusercontent.com/a2o/puppet-modules-a2o-essential/ 9e48057cc1320de52548ff019352299bc4bd5069/modules/a2o_essential_linux_mysql/files/my.cnf. (March 2017).

2017. Stack Overflow. http://stackoverflow.com/. (March 2017).

2017c. Stats-analysis. https://raw.githubusercontent.com/NCIP/stats-analysis/ec7a1a15b0a5a7518a061aedd2d601ea7cc2dfca/ cacoresdk203.2.1/conf/download/my.cnf. (March 2017).

2017a. Stats-analysis. https:/raw.githubusercontent.com/NCIP/stats-analysis/ec7a1a15b0a5a7518a061aedd2d601ea7cc2dfca/ cacoresdk203.2.1/conf/download/my.cnf. (March 2017).

2017. The issue for slow query log. http://forum.directadmin.com/showthread.php?t=47547. (March 2017).

2017d. Type Error Example. https://github.com/thekad/puppet-module-mysql/blob/master/templates/my.cnf.erb. (March 2017).

2017b. vit-analysis. https:/www.dropbox.com/s/09joln8kacu9ceq/ekqjat6m1j5nv9ihjhua9q89sid77cso.cnf?dl=00. (March 2017).

2017c. vitroot. https://raw.githubusercontent.com/vitroot/configs/90441204dbae37521912eaaeedd3574db07b8ae4/my.cnf. (March 2017).

2017d. vitroot2. https://www.dropbox.com/s/qcfmsx12i4pjjtd/missing.cnf?dl=0. (March 2017).

2017c. vps. https://raw.githubusercontent.com/rarescosma/vps/7d0b898bb30eecac65158f704b43bb4d1ca06dbe/_config/ mysql/my.cnf. (March 2017).

Rakesh Agrawal, Tomasz Imieliński, and Arun Swami. 1993. Mining association rules between sets of items in large databases. In Acm sigmod record, Vol. 22. ACM, 207-216.

Mona Attariyan, Michael Chow, and Jason Flinn. 2012. X-ray: Automating root-cause diagnosis of performance anomalies in production software. In 10th USENIX Symposium on Operating Systems Design and Implementation (OSDI).

Mona Attariyan and Jason Flinn. 2010. Automating configuration troubleshooting with dynamic information flow analysis. In 9th USENIX Symposium on Operating Systems Design and Implementation (OSDI).

Jay Ayres, Jason Flannick, Johannes Gehrke, and Tomi Yiu. 2002. Sequential pattern mining using a bitmap representation. In Proceedings of the eighth ACM SIGKDD international conference on Knowledge discovery and data mining. ACM, 429-435.

François Bobot, Jean-Christophe Filliâtre, Claude Marché, and Andrei Paskevich. 2015. Let's verify this with Why3. STTT 17, 6 (2015), 709-727.

Ali Breland. 2017. FCC: Over 12,000 callers couldnâĂŹt reach 911 during AT\&T outage. http://thehill.com/policy/technology/325510-over-12000-callers-couldnt-reach-911-during-att-outage. (March 2017).

Xu Chen, Yun Mao, Zhuoqing Morley Mao, and Jacobus E. van der Merwe. 2010. Declarative configuration management for complex and dynamic networks. In ACM CoNEXT (CoNEXT).

William Enck, Patrick Drew McDaniel, Subhabrata Sen, Panagiotis Sebos, Sylke Spoerel, Albert G. Greenberg, Sanjay G. Rao, and William Aiello. 2007. Configuration Management at Massive Scale: System Design and Experience. In USENIX Annual Technical Conference (USENIX ATC).

Jiawei Han, Hong Cheng, Dong Xin, and Xifeng Yan. 2007. Frequent pattern mining: current status and future directions Data Mining and Knowledge Discovery 15, 1 (2007), 55-86.

Peng Huang, William J. Bolosky, Abhishek Singh, and Yuanyuan Zhou. 2015. ConfValley: A systematic configuration validation framework for cloud services. In 10th European Conference on Computer Systems (EuroSys).

Andrei Nikolaevich Kolmogorov. 1965. Three approaches to the definition of the concept âĂIJquantity of informationâĂ Problemy peredachi informatsii 1, 1 (1965), 3-11.

Pat Langley and Herbert A Simon. 1995. Applications of machine learning and rule induction. Commun. ACM 38, 11 (1995), 54-64.

Tao Lei, Regina Barzilay, and Tommi Jaakkola. 2016. Rationalizing neural predictions. arXiv preprint arXiv:1606.04155 (2016).

K. Rustan M. Leino. 2010. Dafny: An Automatic Program Verifier for Functional Correctness. In Logic for Programming, Artificial Intelligence, and Reasoning - 16th International Conference, LPAR-16. 348-370.

Boon Thau Loo, Joseph M. Hellerstein, Ion Stoica, and Raghu Ramakrishnan. 2005. Declarative routing: Extensible routing with declarative queries. In ACM SIGCOMM (SIGCOMM). 
Nizar R Mabroukeh and Christie I Ezeife. 2010. A taxonomy of sequential pattern mining algorithms. ACM Computing Surveys (CSUR) 43, 1 (2010), 3.

Ruzica Piskac, Thomas Wies, and Damien Zufferey. 2014. GRASShopper - Complete Heap Verification with Mixed Specifications. In Tools and Algorithms for the Construction and Analysis of Systems - 20th International Conference, TACAS 2014. 124-139.

Veselin Raychev, Martin Vechev, and Andreas Krause. 2015. Predicting program properties from big code. In ACM SIGPLAN Notices, Vol. 50. ACM, 111-124.

Jenni Ryall. 2015. Facebook, Tinder, Instagram suffer widespread issues. http://mashable.com/2015/01/27/ facebook-tinder-instagram-issues/. (2015).

Mark Santolucito, Ennan Zhai, and Ruzica Piskac. 2016. Probabilistic Automated Language Learning for Configuration Files. In 28th Computer Aided Verification (CAV).

Ya-Yunn Su, Mona Attariyan, and Jason Flinn. 2007. AutoBash: Improving configuration management with operating systems. In 21st ACM Symposium on Operating Systems Principles (SOSP).

Helen J. Wang, John C. Platt, Yu Chen, Ruyun Zhang, and Yi-Min Wang. 2004. Automatic misconfiguration troubleshooting with PeerPressure. In 6th USENIX Symposium on Operating Systems Design and Implementation (OSDI).

Andrew Whitaker, Richard S. Cox, and Steven D. Gribble. 2004. Configuration debugging as search: Finding the needle in the haystack. In 6th USENIX Symposium on Operating Systems Design and Implementation (OSDI).

Tianyin Xu. 2017. Misconfiguration dataset. https:/github.com/tianyin/configuration_datasets. (March 2017).

Tianyin Xu, Long Jin, Xuepeng Fan, Yuanyuan Zhou, Shankar Pasupathy, and Rukma Talwadker. 2015. Hey, you have given me too many knobs!: understanding and dealing with over-designed configuration in system software. In 10th foint Meeting on Foundations of Software Engineering (ESEC/FSE).

Tianyin Xu, Xinxin Jin, Peng Huang, Yuanyuan Zhou, Shan Lu, Long Jin, and Shankar Pasupathy. 2016. Early detection of configuration errors to reduce failure damage. In 12th USENIX Symposium on Operating Systems Design and Implementation (OSDI).

Tianyin Xu, Jiaqi Zhang, Peng Huang, Jing Zheng, Tianwei Sheng, Ding Yuan, Yuanyuan Zhou, and Shankar Pasupathy. 2013. Do not blame users for misconfigurations. In 24th ACM Symposium on Operating Systems Principles (SOSP).

Tianyin Xu and Yuanyuan Zhou. 2015. Systems approaches to tackling configuration errors: A survey. ACM Comput. Surv. 47, 4 (2015), 70.

Zuoning Yin, Xiao Ma, Jing Zheng, Yuanyuan Zhou, Lakshmi N. Bairavasundaram, and Shankar Pasupathy. 2011. An empirical study on configuration errors in commercial and open source systems. In 23rd ACM Symposium on Operating Systems Principles (SOSP).

Ding Yuan, Yinglian Xie, Rina Panigrahy, Junfeng Yang, Chad Verbowski, and Arunvijay Kumar. 2011. Context-based online configuration-error detection. In USENIX Annual Technical Conference (USENIX ATC).

Andreas Zeller. 2005. Why Programs Fail: A Guide to Systematic Debugging. Morgan Kaufmann Publishers Inc., San Francisco, CA, USA.

Jiaqi Zhang, Lakshminarayanan Renganarayana, Xiaolan Zhang, Niyu Ge, Vasanth Bala, Tianyin Xu, and Yuanyuan Zhou. 2014. EnCore: Exploiting system environment and correlation information for misconfiguration detection. In Architectural Support for Programming Languages and Operating Systems (ASPLOS). 